\title{
Editorial
}

\section{Structural, Electronic, and Optical Properties of Functional Metal Oxides}

\author{
Jianhua Hao, ${ }^{1}$ Nian X. Sun, ${ }^{2}$ Jianrong Qiu, ${ }^{3}$ and Danyang Wang ${ }^{4}$ \\ ${ }^{1}$ Department of Applied Physics, The Hong Kong Polytechnic University, Hong Kong \\ ${ }^{2}$ Electrical and Computer Engineering Department, Northeastern University, Boston, MA, USA \\ ${ }^{3}$ State Key Laboratory of Luminescent Materials and Devices, South China University of Technology, Guangzhou, China \\ ${ }^{4}$ School of Materials Science and Engineering, The University of New South Wales, Sydney, NSW, Australia \\ Correspondence should be addressed to Jianhua Hao; jh.hao@polyu.edu.hk
}

Received 21 July 2014; Accepted 21 July 2014; Published 31 August 2014

Copyright (C) 2014 Jianhua Hao et al. This is an open access article distributed under the Creative Commons Attribution License, which permits unrestricted use, distribution, and reproduction in any medium, provided the original work is properly cited.

In the past few decades, metal-oxides have drawn considerable attention and have been applied in diverse fields of applications because of their full spectrum of various behaviors, such as dielectrics, ferroelectricity, magnetism, superconductivity, optical spectroscopy, and light emission. The research interest in functional metal oxides is reflected by the rapid growth of publications in this field. Many metaloxide materials are usually wideband gap compounds. The conductivity of oxides can vary from insulator to semiconductor to conductor. Due to their varied electronic and optical properties, metal oxides have been used for a wide variety of applications, such as smart sensor, data storage, energy, catalysis, and optical and display devices. The key to these device applications is an improved understanding of fundamental structural, electronic, dielectric, optical, and luminescent properties. In this special issue, we have invited authors to submit original research and review articles that will stimulate the continuing efforts to investigate the basic physical properties.

One of the papers of this special issue investigates lanthanide codoped oxide $\mathrm{Y}_{2} \mathrm{O}_{3}$ with body-centered cubic structure. The oxide nanoparticles exhibit strong red and light yellow upconversion emissions. It is expected that these oxide materials with tunable multicolor output and intense red upconversion emission may have potential application in color displays and biolabels. Other papers present the studies on the typical oxide superconductors of $\mathrm{YBa}_{2} \mathrm{Cu}_{3} \mathrm{O}_{7-x}$ (YBCO). The effects of the sintering temperature on the microstructure and oriented growth of the YBCO film are discussed. The impact of the surface morphologies of buffer layers on the superconducting properties in YBCO films is investigated. Another paper of this special issue presents nice work on the synthesis of $\mathrm{KNbO}_{3}$ nanowires with perovskite structures by hydrothermal treatment at low temperature. The phase transition and photoluminescence (PL) properties of $\mathrm{KNbO}_{3}$ nanowires are investigated. The studies completed by the researchers in China show that the $\mathrm{KNbO}_{3}$ nanowires may be a promising candidate for fabricating ferroelectric and piezoelectric nanodevices.

One paper has examined the local structure and disorder in promising lead-free piezoelectric ceramic systems of NBTBT and KNN. The studies reported by the Australian National University and University of Cambridge may supply information about the origins of high electrostrictive response and antiferroelectric behavior. Another paper reports the synthesis and characterization of nanocrystallite monoclinic zirconia powder. This work performed by the researchers in India will be useful in the applications of high- $\kappa$ gate dielectric materials. Other papers report the synthesis and luminescent characteristics of various types of rare-earth doped oxidebased nanomaterials. These reported materials have potential applications in many fields such as lighting and optoelectronic devices with nanometer dimensions.

Another paper is a review article written by J. G. Reynolds and C. L. Reynolds in North Carolina State University. This review summarizes recent reports on the p-type behavior of $\mathrm{ZnO}$ for the various potential doping schemes. It is suggested that an understanding of the reaction pathways and specific 
model to explain the acceptor level is the key to stable and reproducible p-type $\mathrm{ZnO}$. Some papers have focused on the fabrication, crystal structural, and electrical properties of several kinds of dielectric ceramics based on oxides. One paper performs the simulation and analysis of $\mathrm{GaN}$ wafer bowing on sapphire substrate. The bowing deformation of the wafer on sapphire substrate is studied using finite element analysis software. Another paper reports on the nanoscale characterization of the morphology, polarization switching, and local piezoresponse hysteresis loops for ferroelectric PVDFTrFE film.

We hope that the publication of this special issue will be of reference value for readers to understand the current status and future research direction in the research field on metal oxides materials and devices.

Jianhua Hao

Nian X. Sun

Jianrong Qiu

Danyang Wang 

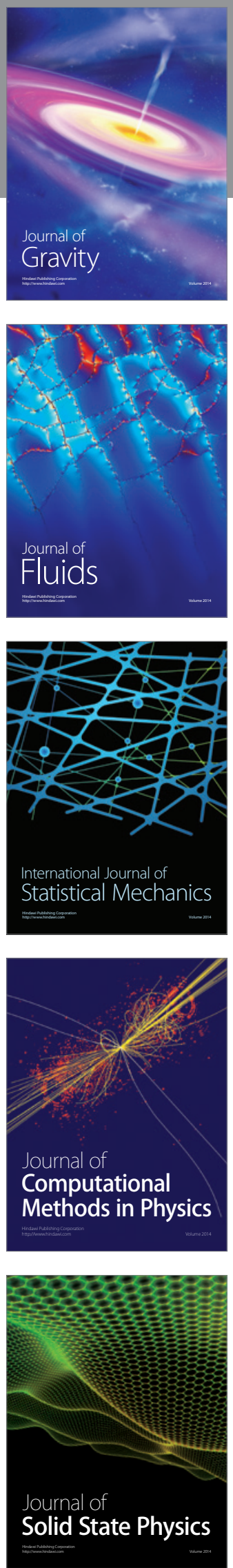

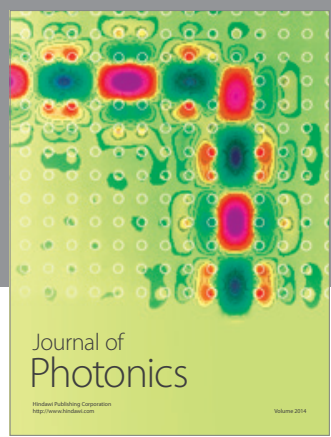

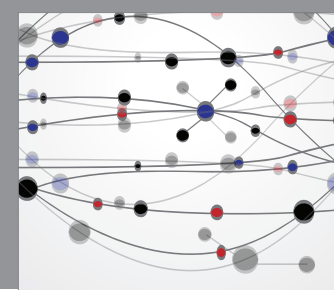

The Scientific World Journal

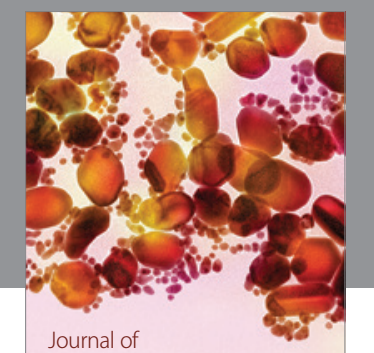

Soft Matter
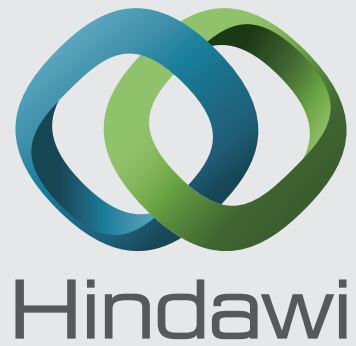

Submit your manuscripts at

http://www.hindawi.com
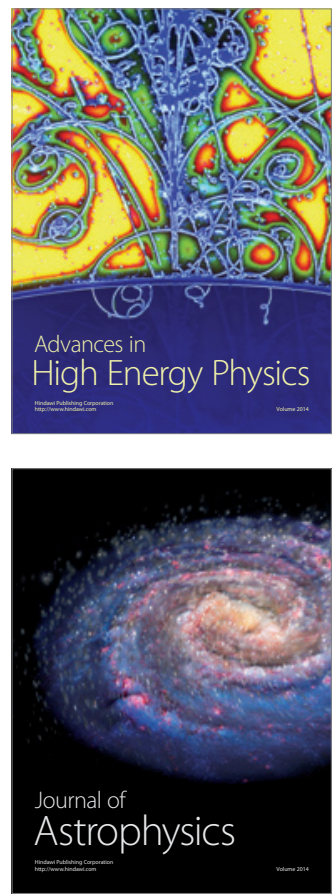
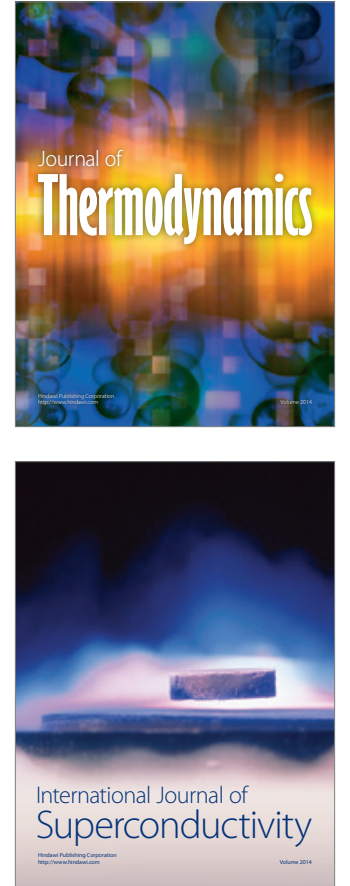
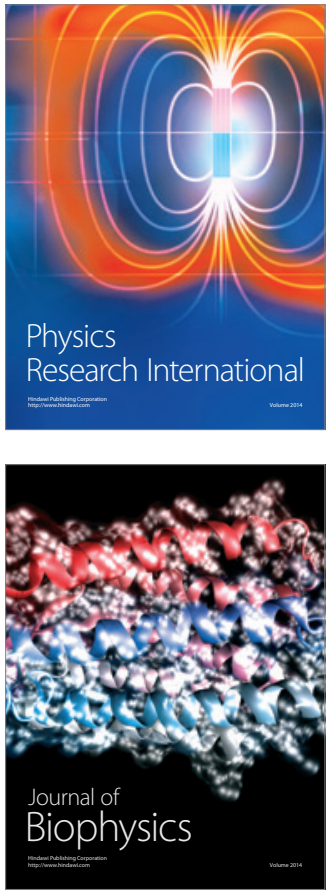
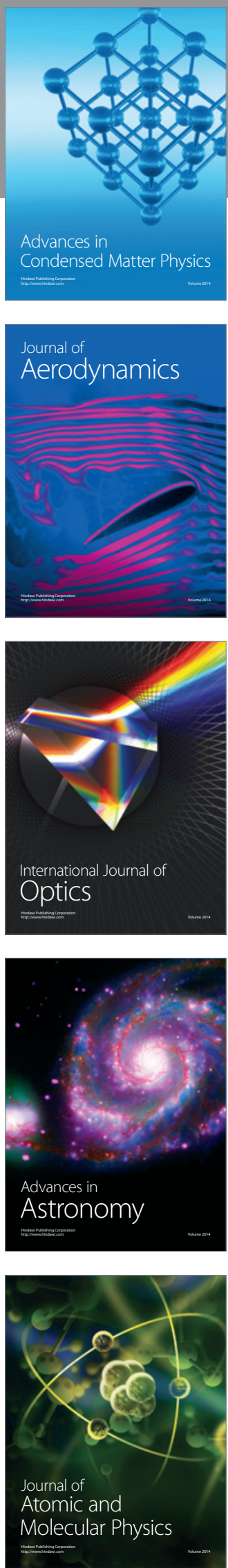